\title{
Mechanisms in Medicine
}

\author{
Donald Gillies ${ }^{1}$
}

Received: 12 May 2017/Accepted: 28 June 2017/Published online: 3 July 2017

(C) The Author(s) 2017. This article is an open access publication

\begin{abstract}
This paper begins by developing a causal theory of mechanisms in medicine, and illustrates the theory with the example of the mechanism of the disease anthrax as elucidated by Koch. The causal approach to mechanisms is then compared to the Machamer, Darden, Craver (MDC) approach. At first sight the two approaches appear to be very different, but it is argued that the divergence is less than it initially seems. There are some differences, however, and it is argued that, where these differences occur, the causal approach to mechanisms is superior.
\end{abstract}

Keywords Causality $\cdot$ Mechanisms $\cdot$ Activities · Intervention · Manipulation

\section{Introduction}

Medicine deals with hypotheses such as (1) A causes D, where D is a disease, or (2) B prevents D, or (3) C cures D. In this paper, I will, for ease of exposition, focus on (1), but the results obtained can easily be extended to (2) and (3) since 'prevents' and 'cures' are both causal notions.

When dealing with a hypothesis such as 'A causes D' it is usually desirable to try to discover a mechanism $M$ which links $A$ to $D$. If the existence of such a mechanism can be established, then $M$ will provide an explanation of the causal hypothesis. The aim of this paper is to give a general account of the nature of these mechanisms, which are to be found in medicine. The approach adopted is that of providing a causal theory of mechanisms in medicine. This causal theory is

Donald Gillies

donald.gillies@ucl.ac.uk

1 Department of Science and Technology Studies, University College London, Gower Street, London WC1E 6BT, UK 
expounded in Sect. 2, and it is illustrated in Sect. 3 by the mechanism of the disease anthrax.

Mechanisms have been much discussed by philosophers of science in the last two or three decades. The most successful approach to mechanisms has been that of Machamer, Darden, Craver (MDC) developed in their 2000 paper. Now the causal theory of mechanisms given in Sects. 2 and 3 appears at first sight to differ considerably from the MDC theory. It is therefore desirable to compare the two approaches, and this is done in the last two sections of the paper. Section 4 gives an exposition of the MDC theory, while Sect. 5 shows how it relates to the causal theory of mechanisms. It turns out that the approaches differ less than might initially be thought, but that they do differ in some respects. Where they differ, it is argued that the causal theory of mechanisms is preferable. The MDC theory made use of some ideas of Anscombe's about causality, and these are also discussed in Sect. 5.

\section{A Causal Theory of Mechanisms}

Let us start then with a causal hypothesis in medicine (H say) of the form A causes $\mathrm{D}$, where $\mathrm{D}$ is some disease. For convenience, I will write A causes D as A $\rightarrow$ D. When handling hypotheses like $\mathrm{H}$, it is often very useful, if not essential to devise a linking mechanism $\mathrm{M}$, such that $\mathrm{A} \rightarrow \mathrm{M} \rightarrow \mathrm{D}$, and to test whether the existence of $\mathrm{M}$ is confirmed or disconfirmed by evidence. Let us make the distinction between basic mechanisms, and mechanisms in general. I will start by considering basic mechanisms, and then introduce mechanisms in general. A basic mechanism $\mathrm{M}$ is defined to be a sequence of causes $\mathrm{C}_{1} \rightarrow \mathrm{C}_{2} \rightarrow \mathrm{C}_{3} \rightarrow \cdots \rightarrow \mathrm{C}_{\mathrm{n}}$ which describe some biochemical/physiological processes going on in the body. A mechanism in general is produced by connecting basic mechanisms together to form a causal network. A complicated branching network of causes may indeed be necessary in some cases to describe a mechanism in medicine. However, the simple linear sequence is surprisingly effective, and is used in the two main examples of mechanisms given in this paper.

There are two further points I would like to make about this approach to mechanisms in medicine. It applies to physical illnesses, but is more problematic for mental illnesses. It is possible that mental disturbances could all be explained in terms of material processes going on in the brain. However, despite the advances of neuroscience, we are very far from being able to give satisfactory explanations of this sort in all cases. The mind-body problem is still an enigma, and to explain psychiatric illnesses, recourse is often made to psychological principles, or to sociological considerations. In this paper, however, I will focus on physical illnesses and will leave aside the tricky problems of psychiatry. Thus my account of mechanisms is limited not just to medicine, but more narrowly to physical illnesses.

Secondly, it should be stressed that when a causal sequence such as $\mathrm{C}_{1} \rightarrow \mathrm{C}_{2} \rightarrow \mathrm{C}_{3} \rightarrow \cdots \rightarrow \mathrm{C}_{\mathrm{n}}$ is given, it is nearly always possible to make the mechanism more detailed by inserting further causal sequences between any two successive causes in the sequence. For instance we could insert a sequence such as $\mathrm{C}_{1}^{\prime} \rightarrow \mathrm{C}_{2}^{\prime} \rightarrow \mathrm{C}_{3}^{\prime}$ between say $\mathrm{C}_{2}$ and $\mathrm{C}_{3}$. This indeed often happens in practice by 
going from one level to a lower level-for example, from the level of bacilli to the level of their DNA or of receptors on their surface. It is usually a matter requiring skilled judgement to decide how detailed a mechanism should be. One needs a mechanism, which is sufficiently detailed for the purposes in hand, without being overloaded with unnecessary complications. The general point here was well expressed by Venn, when he suggested in 1866 that the phrase 'chain of causation' could usefully be replaced with 'rope of causation' (quoted from Salmon 1998, p. 224). Really causal processes are continuous like a rope, and whenever we reduce them to a chain, more causes can usually be inserted between any two links in the chain.

In our causal theory of mechanisms in medicine, mechanisms are defined in terms of causes, but what account is then to be given of causes? My answer is that causes should be characterised by what I propose to call an AIM theory of causality where AIM stands for Action, Intervention, Manipulation. AIM theories are those, which emphasize the links between causality and action, intervention, manipulation. One of the first to develop a detailed version of such a theory of causality in the twentieth century was Collingwood, in his (1938) paper, and subsequent book (1940). Collingwood distinguishes three senses of causality, and his AIM account is applied only to causality in sense II. In this sense, what is caused is an event in nature. However, Collingwood goes on to say (1938, p. 89):

In sense II ... the word cause expresses an idea relative to human action; but the action .... is an action intended to control ... things in 'nature', or 'physical' things. In this sense, the 'cause' of an event in nature is the handle, so to speak, by which we can manipulate it. ...

This sense of the word may be defined as follows. A cause is an event or state of things which it is in our power to produce or prevent, and by producing or preventing which we can produce or prevent that whose cause it is said to be.

Here Collingwood relates cause to action, and introduces the striking comparison of a cause to a handle by which we can manipulate the effect of the cause. He also mentions two kinds of action which can be based on a causal law of the form A causes D. We can either try to produce D (a productive action) or we can try to prevent $\mathrm{D}$ (an avoidance action). Now for medicine avoidance actions are obviously more important, since the aim of medicine is to prevent or cure diseases, not to produce them.

There have been a number of recent developments of AIM theories of causality by philosophers of science. Menzies and Price have developed one of these theories (see Price 1992; Menzies and Price 1993). They refer to their theory as an agency theory of causality. Woodward (2003) has developed a theory, which he calls (p. v): "a manipulationist or interventionist account of ... causation." I refer to my own version (Gillies 2005) as an action-related theory of causality. Pearl's (2000) book should also be mentioned here. Pearl is not committed exclusively to an AIM account of causality, and introduces other conceptions of causality into his scheme. However, intervention still plays an important role for him.

As the previous paragraph shows, the various contemporary theories all have different names, but they are all related to one another and also to the view 
originally developed by Collingwood. To bring out this family resemblance, I am suggesting using the term AIM (Action, Intervention, Manipulation) to describe this group of theories. There is, however, one important difference between Woodward's version of the AIM approach and the rest. All the others take the various actions, interventions, and manipulations to be carried out by human beings, to be human actions. Collingwood, in the quotation just given, makes this explicit. Woodward, however, gives a definition of intervention, which implies that interventions need not be human actions (Woodward 2003, p. 103). Woodward's motivation here is to make the notion of cause less anthropomorphic; but other exponents of the AIM approach do not go along with Woodward here. For example, Buzzoni writes (2014, p. 377):

In spite of the unquestionable merits of Woodward's theory, I cannot agree with its separating the notion of causality from that of human intervention. On this point, I side with von Wright, Price, and Menzies: the close link between intervention and causality cannot be understood without reference to the free agency of human beings.

I agree with Buzzoni on this point, and so, for the rest of the paper, will take an AIM theory to be one in which the actions, interventions, and manipulations are all human actions.

\section{Illustrative Example: The Mechanism of Anthrax}

Anthrax is mainly a disease of animals-particularly cows and sheep, but it can affect humans as well, mainly those who came into contact with infected animals such as shepherds and butchers. Deaths of livestock from anthrax were very common in the 1860s, and the disease led to considerable economic losses to farmers. There was thus a strong motivation to seek for a way of curing or preventing the disease.

Davaine in France examined the blood of animals infected by anthrax under a microscope and found that it contained rod-shaped micro-organisms which he named 'bacteridia'. In 1863 he published a paper in which he postulated that these bacteridia caused anthrax. However, his theory was not well-received. It was strongly criticized by two other scientists (Jaillard and Leplat) in 1865, and did not find general acceptance.

Despite this set-back, anthrax proved to be a very suitable disease for the development of the germ theory. Since it was a disease of both humans and animals, it was easy to carry out experiments on animals, while still dealing with a human disease. Moreover the bacterium involved, Davaine's bacteridium, now called bacillus anthracis, is one of the biggest of the pathogenic bacteria in both length and thickness, and so is easy to study under the microscope. Pasteur in France and Koch in Germany took up Davaine's hypothesis, and their further investigations established that it was correct.

My first example of a mechanism in medicine is based on the treatment of anthrax which is given by Koch in his 1876, 1878, and 1881 papers. Although 
anthrax is a disease which affects both animals and humans, it will be convenient to consider anthrax in sheep. It had been observed by farmers that if sheep grazed in particular fields which they called 'anthrax fields', then many would contract anthrax and die of it. There was thus an empirical causal law of the form:

Sheep grazing in anthrax fields $\rightarrow$ some of them to die with symptoms of anthrax.

Koch's first great discovery regarding anthrax was that the bacillus can form spores which are very resistant to destruction, but which can turn back into bacilli when they enter an animal's blood stream. He therefore postulated that what farmers called 'anthrax fields' were in fact fields which contained many anthrax spores. He further postulated that the spores had got there from (1881, p. 59) "the bloody refuse of sick and dying animals" which had earlier contracted anthrax. Thus he replaced the farmers' empirical causal law just given by the law:

Sheep grazing in fields where there are many anthrax spores $\rightarrow$ some of them to die with symptoms of anthrax.

Koch then connected the cause and effect in this law with the following linking mechanism (M1)

1. Anthrax spores enter the blood stream of some of the sheep through abrasions on their body or via the intestine $\rightarrow$

2. Spores in the blood stream to turn back into anthrax bacilli $\rightarrow$

3. These anthrax bacilli to multiply rapidly $\rightarrow$

4. Anthrax bacilli to enter the capillaries $\rightarrow$

5. Almost all the capillaries in the lungs, liver, kidneys, spleen, intestines, and stomach to be filled with enormous numbers of anthrax bacilli

Koch in the papers just cited gives details of numerous experiments which he carried out, and whose results supported each step of the above mechanism.

That concludes my account of the causal theory of mechanisms. I will now compare this approach to mechanisms to other contemporary treatments of mechanisms.

\section{The Machamer, Darden, Craver (MDC) Approach to Mechanisms}

Salmon devoted considerable time and effort to the study of mechanisms in relation to causality (see his 1994). However, his approach seemed to subsequent philosophers of science too closely related to physics, and so less appropriate for other areas of science, particularly the biological sciences and medicine. Machamer, Darden and Craver have this opinion of Salmon, for they say (2000, p. 7):

Salmon identifies interactions ... more recently, in terms of exchanges of conserved quantities .... Although we acknowledge the possibility that Salmon's analysis may be all there is to certain fundamental types of 
interactions in physics, his analysis is silent as to the character of the productivity in the activities investigated by many other sciences. ... As our examples will show, much of what neurobiologists and molecular biologists do should be seen as an effort to understand these diverse kinds of production and the ways that they work.

So Machamer, Darden and Craver try to give an account of mechanism which applies, not just to physics, but to other sciences as well. As they say (2000, p. 2):

Our goal is to sketch a mechanistic approach for analysing neurobiology and molecular biology that is grounded in the details of scientific practice, an approach that may well apply to other scientific fields.

Their definition of mechanism is the following (2000, p. 3):

Mechanisms are entities and activities organized such that they are productive of regular changes from start or set-up to finish or termination conditions.

They particularly stress the importance of having activities as well as entities in a satisfactory account of mechanisms. The role of activities is described as follows (2000, p. 6):

Activities are types of causes. Terms like "cause" and "interact" are abstract terms that need to be specified with a type of activity and are often so specified in typical scientific discourse. Anscombe ... 1971 ... noted that the word "cause" itself is highly general and only becomes meaningful when filled out by other, more specific, causal verbs, e.g. scrape, push, dry, carry, eat, burn, knock over. An entity acts as a cause when it engages in a productive activity.

While traditionally philosophers have tried to analyse parts of science using the general and abstract term 'cause', Machamer, Darden and Craver suggest that we should instead, in each particular scientific case, try to specify a type of activity and use this activity in our analysis. This is analogous to Anscombe's consideration of specific causal verbs such as scrape, push, etc., though Anscombe here gives examples from everyday life rather than science. Machamer, Darden and Craver then go on, in the rest of their paper, to illustrate this approach by analysing in detail a number of examples from the biological sciences, including (2000, Section 6, pp. 18-21) the discovery of the mechanism of protein synthesis.

The Machamer, Darden and Craver (MDC) approach has become the most popular approach to mechanisms among philosophers of science. Yet it appears to differ very strikingly from the causal approach expounded in Sects. 2 and 3 of this paper. However a more detailed comparison of the two approaches shows that the difference is really less than it might initially seem. I will undertake this detailed comparison in the next section. 


\section{Causes, Activities, and Anscombe}

In the approach of Machamer, Darden and Craver (henceforth MDC), mechanisms are defined using the term 'activities', but the definition contains no mention of the general concept of cause. Conversely in the causal theory of mechanisms developed above, mechanisms in medicine are defined in terms of the general concept of cause, but there is no mention of the more specific concept of activity. We must now compare the two approaches, and a useful way to begin is to consider some observations made by Darden in her 2013.

Darden argues that causal claims are impoverished compared to claims about the mechanisms operating. As she says (2013, p. 20):

I will argue that within the mechanistic sciences, such as molecular biology and molecular medicine, the claim " $\mathrm{C}$ causes $\mathrm{E}$ " is impoverished compared to the claim that "this mechanism produces this phenomenon." Knowledge of a mechanism in the biological sciences is usually more useful for explanation, prediction, and control than merely being able to label something as a cause.

Later in the paper, she goes on to illustrate this general claim by an example taken from the field of medicine. She writes (2013, p. 26):

One might say "A mutation in the CFTR gene causes the disease cystic fibrosis." But this is an impoverished claim, compared to a description of the myriad mechanisms involved in the etiology of the disease.

This last statement of Darden's seems to me quite correct. Let us consider the general case of A causes D $(\mathrm{A} \rightarrow \mathrm{D})$, where $\mathrm{D}$ is some disease. Suppose now a mechanism $\mathrm{M}$ is discovered linking $\mathrm{A}$ to $\mathrm{D}$. Then the statement $(\mathrm{A} \rightarrow \mathrm{D}) \& \mathrm{M}$ will in general be much more informative and yield more important consequences for medicine than the statement $(\mathrm{A} \rightarrow \mathrm{D})$ on its own. If the mechanism $\mathrm{M}$ is analysed in the MDC fashion, then it will be a statement about activities rather than causes, and so the extra important information will not involve the general concept of cause. Suppose, however, we adopt the causal theory of mechanisms in medicine as developed above, then the linking mechanism will take the form $\mathrm{A} \rightarrow \mathrm{M} \rightarrow \mathrm{D}$, and, if $M$ is a basic mechanism, it will have the form $C_{1} \rightarrow C_{2} \rightarrow C_{3} \rightarrow \cdots \rightarrow C_{n}$. So the new statement with the extra mechanistic information will involve many more instances of the general concept of cause than the original statement A $\rightarrow$ D did.

This is illustrated by the anthrax example given in Sect. 3. The original causal law was

Sheep grazing in anthrax fields $\rightarrow$ some of them to die with symptoms of anthrax

This involved one use of $\rightarrow$. Once the mechanism developed by Koch was inserted, however, the number of uses of $\rightarrow$ increased to 6 .

Naturally the mechanistic additions greatly improved knowledge about anthrax. On the basis of the original causal law, the only step which could be taken to stop sheep getting anthrax was to prevent them from grazing in those fields which had been identified empirically as anthrax fields. Once a single causal link had been 
replaced by a chain of six causal links, it was possible to consider preventing anthrax by intervening on any one of the 6 links. Consider the link between 2 and 3 namely:

2. Spores in the blood stream to turn back into anthrax bacilli $\rightarrow$

3. These anthrax bacilli to multiply rapidly

Pasteur succeeded in blocking the usual causal consequence of 2 by developing a vaccine which used attenuated anthrax bacilli. This primed the immune systems of the animals who received it to enable them to destroy any invading anthrax bacilli quickly, and so to prevent such bacilli multiplying rapidly. Such a method of prevention would have been quite impossible, even unthinkable, on the basis of the original causal law.

I will now show that Darden's example of cystic fibrosis can be analysed along similar lines to the anthrax example.

Darden gives details of several mechanisms which are involved in producing cystic fibrosis. For simplicity, I will discuss just one of these mechanisms, which, according to Darden (2013, p. 28): "occurs in about $90 \%$ of patients with cystic fibrosis in the USA." She describes it as follows (2013, p. 28):

Three bases are deleted in the CFTR gene. During protein synthesis, this deletion results in one missing amino acid: phenylalanine at position 508 (of the 1480 amino acids). Although missing only one amino acid, such Delta F 508 mutant proteins do not fold properly. The misfolded proteins do not implant into the cell membrane to properly transport chloride ions in and out of the cell.

This can easily be translated into my sequence of causes approach along the following lines (M2)

1. Three bases are deleted in CFTR gene $\rightarrow$

2. Protein synthesized to miss one amino acid $\rightarrow$

3. Protein to fold wrongly $\rightarrow$

4. Misfolded protein not to implant into the cell membrane $\rightarrow$

5. Cell membrane not to properly transport chloride ions in and out of the cell

Darden goes on to say (2013, p. 29):

Although some of the protein degrades, some of the misfolded protein remains in the cells. Therapy can be directed to finding drugs that aid in rescuing the undegraded misfolded protein so that is refolds and inserts into the cell membrane and functions (albeit at a reduced level) to transport chloride ions.

Darden is quite correct that the single causal law "A mutation in the CFTR gene causes the disease cystic fibrosis" would, on its own, never have suggested developing the therapy just described. However, if the single causal law is made more detailed by inserting the causal sequence M2 $(1 \rightarrow 2 \rightarrow 3 \rightarrow 4 \rightarrow 5)$, then there is ample justification for developing the therapy. In fact the therapy tries to 
prevent the step from 4 to 5 by getting the misfolded protein to refold in the correct manner.

The analysis just given of Darden's cystic fibrosis example surely shows that the difference between the MDC approach and the causal theory of mechanisms is less than it might appear at first sight. The mechanism M2 does contain both entities and activities. The entity (a CFTR gene missing three bases) has the activity of synthesizing an entity: a protein which misses an amino acid. This has the activity of folding wrongly, and so on. The difference is that the causal theory approach retains the general notion of cause $(\rightarrow)$ which marks each link in the chain, and the entities and activities are confined to the nodes in the network.

Despite this large overlap with the MDC approach, I will next argue that the causal theory of mechanisms is to be preferred to the MDC approach when analysing mechanisms in medicine. There are in fact a number of reasons why the causal theory approach should be judged superior. First of all it is closer to the way that medical researchers analyse mechanisms. What I have called a basic mechanism is more or less the same as what medical researchers call a pathway. Secondly it is useful to have the causal arrows $(\rightarrow)$ because they indicate the points at which it might be possible to intervene in order to avoid an undesirable consequence, or to produce a desirable one. This is in accordance with the AIM (Action, Intention, Manipulation) approach to causality. Thirdly the causal theory of mechanisms in medicine avoids the difficulty for the MDC approach produced by causation by absence. In fact causation by absence is a very common feature of mechanisms in medicine. If a gene is missing, this may mean that a protein is not produced. The absence of this protein may in turn mean that a hormone is not produced, and the absence of this hormone may in turn cause a disease. This is a problem for the MDC approach since, if an absence causes something, there are no entities whose activities produce the result. On the other hand, there is no problem for an absence causing something on the AIM approach to causality. Suppose the causal law is that the absence of hormone $\mathrm{H}$ produces disease $\mathrm{D}$. We can base actions and interventions on such a law just as on any other causal law. For example, to cure $\mathrm{D}$ we have only to supply hormone $\mathrm{H}$, perhaps by means of a pill. This is a typical avoidance action based on a causal law A causes B, where, in order to remove B, we have to ensure that A does not occur. From the point of view of AIM theories of causality, ensuring that an absence does not occur, is no different from ensuring that an undesirable presence does not occur.

I next want to consider the passage from Anscombe cited by MDC. Anscombe's point seems to me correct, but I will argue that it supports the causal approach to mechanisms in medicine (when combined with an AIM theory of causality) better than it supports the MDC approach. The discussion of this will lead to a further, and perhaps the most cogent, reason for preferring the causal approach to mechanisms to the MDC approach. Anscombe says (1971, pp. 92-93):

The truthful ... answer to the question: How did we come by our primary knowledge of causality? is that in learning to speak we learned the linguistic representation and application of a host of causal concepts. Very many of them were represented by transitive and other verbs of action used in reporting 
what is observed. ... The word 'cause' itself is highly general. How does someone show that he has the concept cause? We may wish to say: only by having such a word in his vocabulary. If so, then the manifest possession of the concept presupposes the mastery of much else in language. I mean: the word "cause" can be added to a language in which are already represented many causal concepts. A small selection: scrape, push, wet, carry, eat, burn, knock over, keep off, squash, make (e.g., noises, paper boats), hurt. But if we care to imagine languages in which no special causal concepts are represented, then no description of the use of a word in such languages will be able to present itself as meaning cause.

It is often useful for understanding philosophical texts, to place them in their historical context. The above passage is taken from Anscombe's inaugural lecture as professor of philosophy at Cambridge in 1971. ${ }^{1}$ From the end of the Second World War until the early 1970s, the dominant school of philosophy in the UK was ordinary language philosophy. This was partly based on the later philosophy of Wittgenstein, particularly his Philosophical Investigations (1953). Anscombe had been a student of Wittgenstein, and was the principal translator of his works into English. The other branch of ordinary language philosophy was based in Oxford, and the two major figures there were J.L.Austin and Gilbert Ryle. Anscombe was an Oxford don from 1946 to 1970 before returning to Cambridge as professor, and she was influenced by the Oxford approach to ordinary language philosophy as well as by Wittgenstein.

In 1956, J.L.Austin gave what became a classic statement and defence of ordinary language philosophy in his presidential address to the Aristotelian Society on 29 October. Austin begins his address by arguing that 'excuses' are a good topic for philosophers to study. This particular example leads him on to some general observations about ordinary language philosophy. He says (1956, p. 7):

... the study of excuses may throw light on ethics. But there are also reasons why it is an attractive subject methodologically, at least if we are to proceed from "ordinary language", that is, by examining what we should say when, and so why and what we should mean by it. Perhaps this method, at least as one philosophical method, scarcely requires justification at present- too evidently, there is gold in them thar hills: ... I will, however, justify it briefly.

A key part of Austin's justification of ordinary language philosophy is the following (1956, p. 8):

... our common stock of words embodies all the distinctions men have found worth drawing, and the connexions they have found worth making, in the lifetimes of many generations: these surely are likely to be more numerous, more sound, since they have stood up to the long test of the survival of the fittest, and more subtle, at least in all ordinary and reasonably practical matters, than any that you or I are likely to think up in our armchairs of an afternoon-the most favoured alternative method.

\footnotetext{
${ }^{1}$ I attended this lecture, since I was a research fellow of King's College Cambridge at the time.
} 
Austin goes on to advocate what he calls (1956, p. 9) "field work in philosophy" which consists in studying how we use a whole range of words when discussing a topic in everyday life. He illustrates this idea by considering aesthetics (1956, p. 9):

How much it is to be wished that similar field work will soon be undertaken in, say, aesthetics; if only we could forget for a while about the beautiful and get down instead to the dainty and the dumpy.

Austin here suggests that philosophers studying aesthetics have focussed too much on considering 'the beautiful' and progress would be made by considering more specific terms such as 'the dainty', 'the dumpy' and so on. It is obvious that Anscombe has been influenced by this passage from Austin when she moves from the general word 'cause' to more specific words such as 'scrape', 'push', 'wet', 'carry' and so on. This is not to say that Anscombe is simply a follower of Austin. In order to understand her position, it is necessary to consider not just ordinary language philosophy but also some of its critics.

In the 1950s, ordinary language philosophy was undoubtedly the dominant school of philosophy in the UK, but still there were quite a number of philosophers in the UK at that time who were strongly critical of this approach to philosophy. The most famous of these critics was Bertrand Russell. Russell was born on 18 May 1872, and so was 84 years old when Austin gave his presidential address to the Aristotelian Society. But Russell, despite his age, was still intellectually formidable, and the next year (1957) he published a critique of ordinary language philosophy in Mind. The article was a reply to some criticisms of Russell's theory of descriptions which had been made by an Oxford philosophy don (Peter Strawson). The specifics of this controversy are interesting, but do not concern us here. Russell regarded Strawson as a member of the school of ordinary language philosophy, and, as part of his reply to Strawson gave the following criticism of this approach to philosophy (1957, pp. 387-388):

Everybody admits that physics and chemistry and medicine each require a language which is not that of everyday life. ... Let us take, in illustration, one of the commonest words in everyday speech: namely, the word "day".... Astronomers ... have three sorts of day: the true solar day: the mean solar day; and the sidereal day. These have different uses ... the sidereal day is relevant if you are trying to estimate the influence of the tides in retarding the earth's rotation. ... If astronomers were subject to the prohibition of precision which some recent philosophers apparently favour, the whole science of astronomy would be impossible.

For technical purposes, technical languages differing from those of daily life are indispensable.

Russell clearly intends his criticism to lead to a total rejection of ordinary language philosophy, but, if we compare what he says with what Austin says, a compromise seems possible. Austin defends ordinary language philosophy as "one philosophical method", and specifies that it is to be used in "ordinary and reasonably practical matters", while Russell argues that the approach is inadequate for dealing with "physics and chemistry and medicine". These views are compatible. Ordinary 
language philosophy might be appropriate when dealing with problems of everyday life, but not when dealing with problems of the sciences.

Bearing this in mind, let us look again at the passage from Anscombe quoted above. Anscombe does not say, as a strict follower of Austin might have done, that we should consider special causal concepts such as scrape, push, we, carry, etc. instead of considering the general concept 'cause'. Her point is rather that the introduction of the general concept of cause can only occur in a language which already contains the specific causal concepts. Now this point is correct and very valuable in defending the AIM (Action, Intervention, Manipulation) approach to causality. One problem with this approach is that it appears to involve a vicious circularity. AIM theories of causality characterise causality in terms of human actions, but human actions involve some notion of causality. So there seems to be a circularity here. It is worth noting in this context that all of Anscombe's special causal concepts (scrape, push, wet, carry, ...) can be taken as referring to human actions, though this is not the only interpretation in several cases. If we limit the interpretation to human actions, Anscombe's point is that we cannot introduce the general concept 'cause' unless we have mastered a considerable vocabulary dealing with human actions. To put it another way, the understanding of the general concept of 'cause' as it is used in medicine in cases like 'infection by a papilloma virus causes cervical cancer' presupposes the earlier understanding of concepts describing human actions. To use a Wittgensteinian terminology, which might well have appealed to Anscombe, we can say that this earlier understanding comes from participating in language games which involve words for human actions such as 'scrape', 'push', etc. Given that this is so, it is legitimate to characterise the concept of cause, as used in medicine, in terms of human actions, and thus AIM theories of causality do not involve a vicious circularity. So Anscombe's point provides a good defence of AIM theories of causality against the objection of circularity.

Russell argues that the sciences require technical languages differing from those of daily life. Such technical languages usually involve some mathematics, and mathematics has a characteristic which differentiates it sharply from ordinary language. Ordinary language characteristically has a considerable range of words describing different objects, activities, and qualities. Mathematical systems, by contrast, are usually based on just a few key concepts in terms of which any other concepts used are defined. These are concepts such as natural number, set, probability. Now medicine is not very strongly mathematized, but there is one situation in which mathematics is used. This is in analysing diseases which are produced by several indeterministic causes. The basic technique which has evolved to deal with such cases is that of causal networks, such as the network illustrated in Fig. 1.

In causal networks the arrow connecting two nodes $\mathrm{A}$ and $\mathrm{C}$ say, means that $\mathrm{A}$ causes $\mathrm{C}$ (or more strictly that $\mathrm{A}$ exerts a causal influence on $\mathrm{C}$ ). These networks usually have associated probability distributions. In the network of Fig. 1, the nodes $\{\mathrm{A}, \mathrm{B}, \mathrm{C}, \mathrm{D}, \mathrm{E}\}$ can be regarded as random variables which have a joint probability distribution. In many cases statistics enable estimates to be made of the probabilities involved. Mathematical techniques have been developed which allow probability calculations to be carried out in such causal probability networks, and, because of 


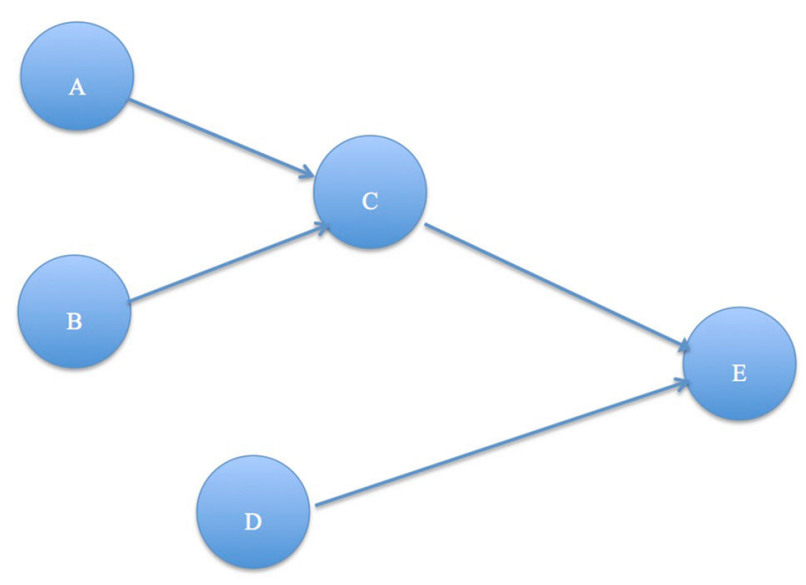

Fig. 1 A typical causal network

their connection with causality, such probabilities can be used to guide actions regarding the cure and prevention of illnesses.

The validity of these mathematical techniques depends, however, on the arrows in the network having a uniform interpretation. Suppose, for example, that the arrow joining $\mathrm{A}$ to $\mathrm{C}$ in Fig. 1 meant that the activity of $\mathrm{A}$ produces $\mathrm{C}$, while the arrow $\mathrm{C}$ to $\mathrm{E}$ meant that the activity of $\mathrm{C}$ produces $\mathrm{E}$, and so on. One would need to take account of all these differences in trying to calculate the effect of $\mathrm{A}, \mathrm{B}$, and $\mathrm{C}$ on $\mathrm{E}$. This would complicate the calculations, if indeed they could be made at all. It is much easier from the mathematical point of view to give the arrows a uniform interpretation throughout. This is my last reason for preferring a causal theory of mechanisms to the MDC approach, and it seems to me the strongest reason.

I can now sum up as follows. Basic mechanisms in medicine are defined as finite linear sequences of causes $\left(\mathrm{C}_{1} \rightarrow \mathrm{C}_{2} \rightarrow \mathrm{C}_{3} \rightarrow \cdots \rightarrow \mathrm{C}_{\mathrm{n}}\right)$, which describe biochem$\mathrm{ical} / \mathrm{physiological}$ processes in the body. This definition corresponds closely to the term 'pathway' often used by medical researchers. Such basic mechanisms can be fitted together to produce more complicated mechanisms which are represented by networks. Entities and activities are often involved, but these are confined to the nodes of the networks, while the arrows of the networks have a uniform causal interpretation in order to facilitate mathematical calculations.

Acknowedgements The research for this paper was performed as part of the project Evaluating Evidence in Medicine (AH/M005917/1), and the author is grateful to the AHRC for supporting this Project. I have had many discussions about mechanisms with the other participants in the Project, notably Brendan Clarke, Phyllis McKay Illari, Veli-Pekka Parkkinen, Federica Russo, Christian Wallmann, Michael Wilde, and Jon Williamson, and many of the points they raised have found their way into the paper. I also had very useful email correspondences with Lindley Darden and Stuart Glennan regarding earlier drafts of some of the material in the paper. These resulted in quite a number of corrections and developments. 
Open Access This article is distributed under the terms of the Creative Commons Attribution 4.0 International License (http://creativecommons.org/licenses/by/4.0/), which permits unrestricted use, distribution, and reproduction in any medium, provided you give appropriate credit to the original author(s) and the source, provide a link to the Creative Commons license, and indicate if changes were made.

\section{References}

Anscombe GEM (1971) Causality and Determination. In: Sosa Ernest, Tooley Michael (eds) Causation, Oxford readings in philosophy. Oxford University Press, Oxford, pp 88-104

Austin JL (1956) A plea for excuses. In: Proceedings of the aristotelian society, 57, pp 1-30

Buzzoni Marco (2014) The agency theory of causality, anthropomorphism, and simultaneity. Int Stud Philos Sci 28(4):375-395

Carter KC (1987) Essays of Robert Koch (Trans: Carter KC). Greenwood Press

Collingwood RG (1938) On the so-called idea of causation. Proc Aristot Soc 38:85-112

Collingwood RG (1940) An essay on metaphysics. Clarendon Press, Oxford, pp 285-337

Darden L (2013) Mechanisms versus causes in biology and medicine. In: Chao H-K et al (eds) Mechanism and causality in biology and economics. Springer, Berlin, pp 19-34

Gillies Donald (2005) An action-related theory of causality. Br J Philos Sci 56:823-842

Koch R (1876) The etiology of anthrax, founded on the course of development of the Bacillus Anthracis (Trans: Carter, 1987), pp 1-17

Koch R (1878) Investigations of the etiology of wound infections (Trans: Carter, 1987), pp 19-56

Koch R (1881) On the etiology of anthrax (Trans: Carter, 1987), pp. 57-81

Machamer P, Darden L, Craver CF (2000) Thinking about Mechanisms. Philos Sci 67(1):1-25

Menzies P, Price H (1993) Causation as a secondary quality. Br J Philos Sci 44:187-204

Pearl Judea (2000) Causality. Models reasoning and inference. Cambridge University Press, Cambridge Price H (1992) Agency and causal asymmetry. Mind 101:501-520

Russell B (1957) Mr. Strawson on referring. Mind 66:385-389

Salmon W (1994) Causality without counterfactuals. Reprinted in Salmon 1998, pp 248-260

Salmon W (1998) Causality and explanation. Oxford University Press, Oxford

Wittgenstein L (1953) Philosophical investigations. Blackwell, Oxford

Woodward J (2003) Making things happen: a theory of causal explanation, Paperback edn. Oxford University Press, Oxford 